\title{
Linx
}

Revue des linguistes de l'université Paris X Nanterre

\section{El fansubbing de locuciones y paremias del español al chino: el caso de la serie de televisión española $E l$ Ministerio del Tiempo}

Peng Gao

\author{
(2) OpenEdition \\ Journals \\ Edición electrónica \\ URL: http://journals.openedition.org/linx/3843 \\ DOI: $10.4000 / \operatorname{linx} .3843$ \\ ISSN: 2118-9692 \\ Editor \\ Presses universitaires de Paris Nanterre
}

Referencia electrónica

Peng Gao, «El fansubbing de locuciones y paremias del español al chino: el caso de la serie de televisión española El Ministerio del Tiempo », Linx [En ligne], 13 | 2020, mis en ligne le 30 mai 2020, consulté le 01 juin 2020. URL : http://journals.openedition.org/linx/3843 ; DOI : https://doi.org/ $10.4000 / \operatorname{lin} x .3843$

Este documento fue generado automáticamente el 1 junio 2020.

Département de Sciences du langage, Université Paris Ouest 


\section{El fansubbing de locuciones y paremias del español al chino: el caso de la serie de televisión española El Ministerio del Tiempo}

\section{Peng Gao}

\section{Introducción}

\subsection{El Fansubbing en China}

1 El fansubbing (abreviatura de fan-subtitling) es una de las variedades de la traducción no profesional en la Traducción Audiovisual y se refiere a la subtitulación «por fanes y para fanes» (Díaz-Cintas/Muñoz Sánchez 2006). Según Ferrer Simó (2005: 27), los subtituladores que participan en el fansubbing son fansubber(s) mientras que los productos de esta actividad de traducción son fansubs. La definición del fansubbing ha cambiado con el tiempo, por lo que actualmente se usa para referirse a la subtitulación no profesional de todo tipo de productos audiovisuales.

En la última década, este fenómeno ha aumentado notablemente en China, como demuestra la gran cantidad de fansubs. Según Cui (2017: 19), los tres grupos de fansubbing más grandes en China (YYeTs, YTET y ShenYing) habían subtitulado hasta el año 2017 un total de 19.230 productos de 10 lenguas distintas.

Entre los estudios existentes cabe mencionar el artículo de Wang (2017), que investiga el proceso de trabajo de YYeTs (el grupo de fansubs más influyente en China). Basándose en ejemplos reales, este autor describe dos particularidades (o en sus palabras, «dos aspectos de creatividad») en los fansubs del inglés al chino: las técnicas explicativas y el lenguaje coloquial ( $c f$. Wang 2017: 177-185). Algunas de sus conclusiones se pueden encontrar completa o parcialmente en el fansubbing del español al chino. 
4 En el mercado audiovisual chino es más fácil encontrar películas y series de los países anglófonos que de los países hispanohablantes. En este contexto, los fansubs del español al chino ayudan a introducir los productos audiovisuales en español en el país asiático y proporcionan otra vía para conocer la lengua y cultura española. Así pues, los grupos de fansubbing en China habían traducido hasta diciembre de 2018 un total de 27 series españolas de televisión, entre las que se encuentran La peste y La casa del papel. Entre estos grupos, ShenYing y YYeTs están a la cabeza, con más de la mitad de las series.

5 Igual que en el fansubbing del inglés al chino, los fansubbers del español al chino utilizan con frecuencia notas en la pantalla con el objetivo de facilitar el entendimiento de los referentes culturales, el argumento, etc. Las notas en la pantalla pueden aparecer de tres formas (cf. Wang 2017: 179): ) notas insertas en la línea de los subtítulos traducidos en chino, al lado del objeto que se explica; ) notas en la parte superior de la pantalla, normalmente con color diferente y/o con estilo diferente del fondo de la pantalla; ) notas libres que aparecen en cualquier parte de la pantalla, normalmente con el mismo color o un color similar. Desde el punto de vista de Pérez-González (2007: 75-77) y Wang (2017: 179), las notas del tipo ) se pueden considerar como «texto[s] inserto[s] que se armonizan con su entorno visual». A continuación, mostramos brevemente cómo se utilizan estas notas libres (o «textos insertos» en palabras de Pérez-González 2007 y Wang 2017) en el fansubbing del español al chino y dejamos de lado las notas de tipo i) y ii), puesto que son más fáciles de reconocer.

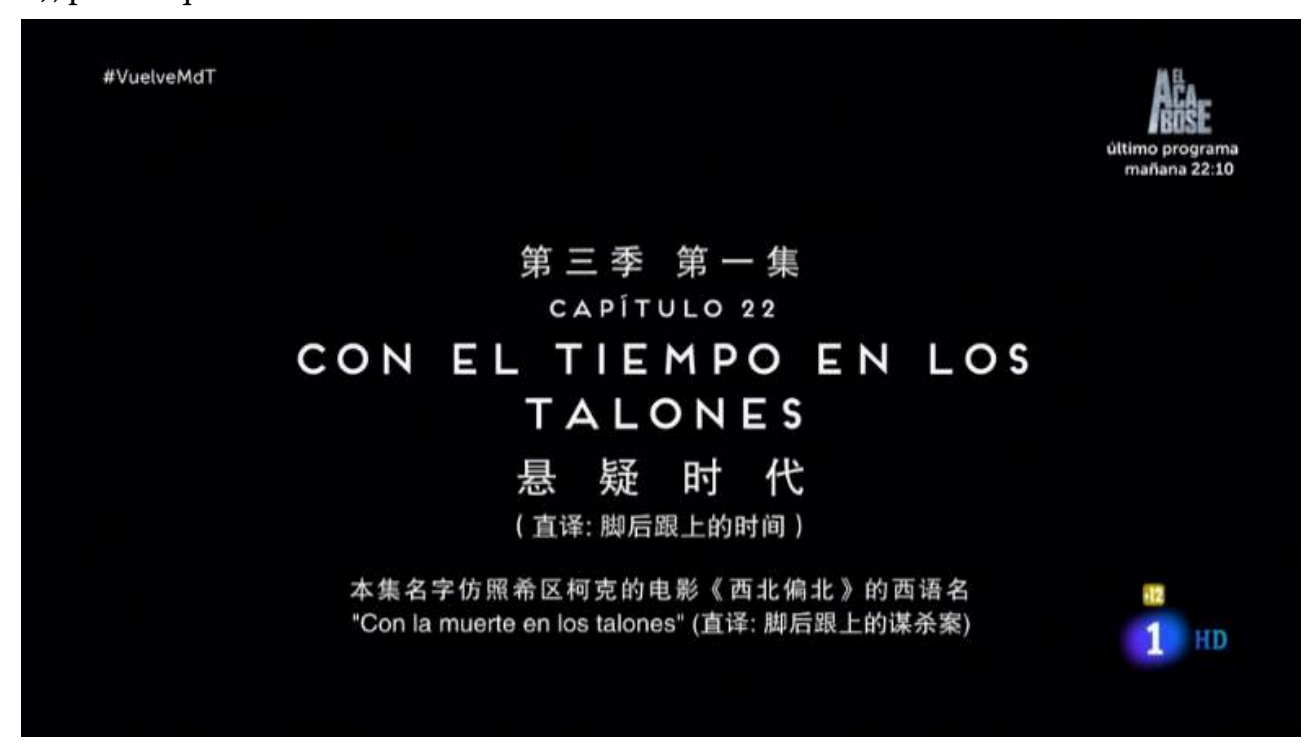

Figura 1: notas libres sobre el título del T3E22 (episodio 22 de la Temporada 3). ShenYing. Captura en $16 / 11 / 2018$

6 La Figura 1 incluye cuatro notas libres: La nota 1 significa «el primer episodio de la temporada 3»; la nota 2 contiene el título inventado del grupo ShenYing para este episodio, llamado «Época de suspense»; la nota 3 indica que la traducción literal del título original de este episodio es «Tiempo en los talones»; y la nota 4 explica que el título original de este episodio remite al título en español de la película de Hitchcock Con la muerte en los talones, proporcionando entre paréntesis la traducción literal del título español de la película, que es «Asesinato en los talones». Mediante estas notas, los espectadores pueden entender la información en la pantalla y ver que este episodio está relacionado con la película de Hitchcock (North by Northwest, 1959). 
7 La Figura 2 se ha extraído del T1E08 (episodio 8 de la temporada 1). Uno de los personajes recibe un mensaje de la antigua funcionaria del Ministerio, Lola Mendieta, justo cuando el director y su equipo están deliberando sobre la traición de esta y el daño que causó al Ministerio. En este contexto es importante que el espectador conozca el remitente del mensaje, hecho que explica la nota en chino «nuevo mensaje de Lola Mendieta». A través de la traducción de la información en la pantalla y la inserción textual, los fansubbers «introducen una dimensión no diegética en el proceso de mediación interlingüística e intercultural» (Pérez-González 2007: 76).

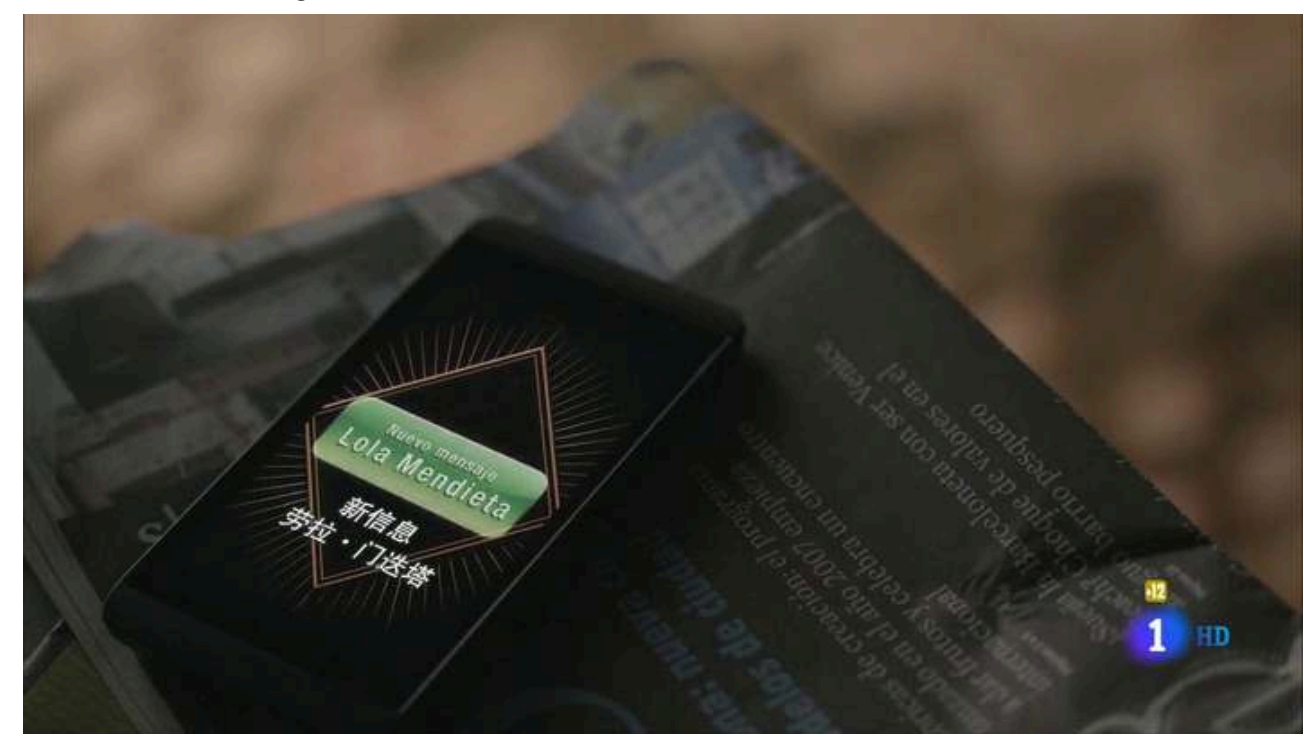

Figura 2: «Nuevo mensaje Lola Mendieta», T1E08. ShenYing. Captura en 16/11/2018

\subsection{La serie de televisión El Ministerio del Tiempo en China}

8 El Ministerio del Tiempo (MdT) es una serie española de televisión del género fantástico y ficción histórica que se emitió entre 2015 y 2017 y que cuenta con tres temporadas y 34 episodios. Aparte de tener una gran audiencia internacional, esta serie también tiene una comunidad de fanes fuerte que se hacen llamar a sí mismos «ministéricos». Esta serie de televisión versa sobre una agencia misteriosa del gobierno. Los agentes del Ministerio del Tiempo deben hacer viajes a diferentes épocas para impedir cualquier cambio posible en la historia de España.

9 Los dos fansubs del MdT en China fueron realizados por los grupos ShenYing y YYeTs. La Tabla 1 muestra la información básica de los dos fansubs. Estas dos traducciones están disponibles en los foros de dichos grupos y en redes sociales como Weibo. Después de la aparición de los fansubs, la televisión nacional de China (CCTV en inglés) importó oficialmente la versión subtitulada de la Temporada 1 en febrero de 2018.

10 Lo más interesante es que los espectadores pueden visualizar estos fansubs en una plataforma china (Bilibili) y publicar su propio «danmu». A diferencia de los comentarios en YouTube, un «danmu» $»^{1}$ es un tipo de comentario sincronizado que se graba con los fotogramas de la serie. Eso permite a diferentes espectadores interactuar simultáneamente sobre cualquier imagen aparecida en la pantalla ( $c f$. Zhang y Cassany 2019). 


\begin{tabular}{|l|l|l|}
\hline & Fansub de ShenYing & Fansub de YYeTs \\
\hline $\begin{array}{l}\text { Fecha de la primera } \\
\text { publicación }\end{array}$ & 27 de septiembre de 2015 & 11 de septiembre de 2016 \\
\hline Episodios traducidos & $\begin{array}{l}\text { T1: } 8 \text { episodios (completo) } \\
\text { T2: } 13 \text { episodios } \\
\text { (completo) } \\
\text { T3: } 13 \text { episodios } \\
\text { (completo) }\end{array}$ & $\begin{array}{l}\text { T1: } 8 \text { episodios (completo) } \\
\text { (incompleto) } \\
\text { T3: } 1 \text { episodios } \\
\text { (incompleto) }\end{array}$ \\
\hline
\end{tabular}

Tabla 1: Los dos fansubs del MdT en China

\section{Marco teórico}

\subsection{Unidades fraseológicas en español: locuciones y paremias}

11 A la hora de hablar de las locuciones y paremias en español, es inevitable enfrentarnos a un conjunto de términos que no han encontrado consenso entre los estudiosos de este ámbito. Este desacuerdo se pone de manifiesto en la existencia de un centenar de denominaciones, como pueden ser «modismo», «expresiones idiomáticas», etc. Puesto que es imposible comparar todas las propuestas definitorias, decidimos seguir la presentada por Corpas Pastor (1997), ampliamente aceptada, y de este modo entendemos que las locuciones y paremias forman parte de las unidades fraseológicas.

Desde el punto de vista de Corpas Pastor (1997: 20), las unidades fraseológicas (UFS) son «unidades léxicas formadas por más de dos palabras gráficas en su límite inferior, cuyo límite superior se sitúa en el nivel de la oración compuesta» y tienen siete características entre las que destacamos la alta frecuencia de uso, la estabilidad y la idiomaticidad (cf. Corpas Pastor 1997: 20-32). Además, Corpas Pastor distingue entre colocaciones, locuciones y enunciados fraseológicos, dividiendo la categoría de los enunciados fraseológicos en paremias y fórmulas rutinarias (cf. Corpas Pastor 1997: 270-271).

13 En el presente artículo abordamos únicamente las locuciones y las paremias por las siguientes razones. Primero, algunos autores como Lawick (2006) consideran las colocaciones como las UFS menos prototípicas; segundo, las fórmulas rutinarias incluyen expresiones como «Hasta luego» $\mathrm{y}$ «Lo siento», que no provocan tantas dificultades a los fansubbers como locuciones y paremias, por lo que no parece imprescindible analizar sus traducciones en el fansubbing. Por último, es imposible analizar de manera exhaustiva y con profundidad todas las UFS que se utilizan en MdT.

14 A continuación, presentamos las definiciones de locuciones y paremias según Corpas Pastor (1997).

Locuciones. «[U]nidades fraseológicas del sistema de la lengua con los siguientes rasgos distintivos: fijación interna, unidad de significado y fijación externa pasemática». Las locuciones «no constituyen enunciados completos, $\mathrm{y}$, generalmente, funcionan como elementos oracionales» (Corpas Pastor 1997:88).

Paremias. Enunciados completos, constituyen actos de habla y presenta fijación interna y externa. Se distingue a las fórmulas rutinarias en dos aspectos 
fundamentales: ) las paremias poseen significado referencial, mientras que el significado de las fórmulas rutinarias es social, expresivo o discursivo; ) las paremias tienen autonomía textual, mientras que las fórmulas dependen de situaciones (cf. Corpas Pastor 1997: 132-133).

Corpas Pastor proporciona una taxonomía de las locuciones y clasifica las paremias en tres tipos. Basándose en su propuesta, Sevilla Muñoz y Crida Álvarez (2013) proponen, a su vez, una taxonomía más detallada de las paremias. No obstante, en esta fase del estudio, no se requiere una clasificación exhaustiva de las locuciones y paremias, por lo que no se tendrá en cuenta estas clasificaciones en el presente artículo.

\subsection{Unidades fraseológicas en chino}

En chino, normalmente se utiliza shuyu para referirse al conjunto de las frases hechas, las locuciones, las paremias, etc. Para el presente estudio usamos la clasificación de Wu (2014: 27), que adapta la propuesta de Sun Weizhang (1989) y la de Corpas Pastor (1997). A su entender, las UFS chinas o shuyu se clasifican en: ) sintagmas fraseológicos, incluyen chengyu y guanyongyu; ) enunciados fraseológicos, engloban xiehouyu, yanyu y geyan. A continuación, presentamos las definiciones de estas UFS del chino con excepción de xiehouyu, dado que no aparece en nuestro corpus.

- Chengyu: es una locución o expresión corta e invariable, normalmente compuesta por cuatro caracteres chinos ${ }^{2}$. Los chengyu se utilizan en la lengua escrita, son «[u]na de las particularidades más notables del idioma chino» $\mathrm{y}$ "presentan con gran fuerza una imagen o un episodio procedente de la literatura, la historia o la tradición popular», se caracterizan por «su rigurosa estructura formal, la mayor frecuencia de uso [...]» (Ramírez Bellerín 2004: 100). Los chengyu corresponden a las frases hechas o las locuciones en la fraseología española.

- Guanyongyu: «es una combinación estable, compuesta por tres o más de tres caracteres, que funciona como elemento oracional y cuyo sentido no es deducible de la suma de los significados de sus componentes. [...]. Se caracteriza por su lenguaje coloquial» (Wu 2014: 56).

- Yanyu y geyan: estas UFS son enunciados completos, se pueden considerar como sentencias y corresponderían a las paremias de las UFS del español. Yanyu son frases breves que se divulgan oralmente y que suelen emplear el lenguaje coloquial debido a su origen popular; geyan son enunciados extraídos de los textos escritos o del habla de personajes conocidos. En ellos se emplea el lenguaje culto o clásico ( $c f$. Wu 2014: 84-86).

\subsection{Las técnicas de traducción de locuciones y paremias}

17 En el presente artículo utilizamos el término «técnica» en lugar de "estrategia» de traducción siguiendo el ejemplo de Hurtado Albir (2001: 249; 266). De este modo, entendemos la técnica de traducción como la aplicación concreta operada por los fansubbers, que afecta a las microunidades textuales de los subtítulos traducidos y es visible en los fansubs. Hasta hoy, los traductólogos todavía no se ponen de acuerdo sobre la clasificación de las técnicas de traducción y se observan variaciones en la terminología empleada. Para facilitar la identificación de las técnicas de traducción, y dado que las locuciones y paremias son objetos de estudio de la fraseología, intentamos adaptar las diferentes propuestas y utilizar una terminología clara sin causar aún más la disparidad. 

investigadores, como la transferencia de los ECRs de Pedersen (2011), las técnicas de traducción de Molina (2006) y la traducción de chengyu de Ramírez Bellerín (2004), elaboramos una lista de las técnicas de traducción de las locuciones y paremias en el fansubbing del español y chino. En la siguiente lista, «UFS» se refiere exclusivamente a las locuciones y paremias, mientras que «No UFS» indica que en español no se usan locuciones o paremias (pero sí que pueden ser colocaciones o fórmulas rutinarias) o que, en chino, las expresiones no son shuyu.

(i) UF $\rightarrow$ UF casi idéntica. Sustitución con una UF casi idéntica del chino. La UF del chino es muy parecida a la UF del español tanto en el significado como en la metáfora/ imagen.

(ii) UF $\rightarrow$ UF diferente. Sustitución con una UF diferente del chino. En este caso, la UF del chino es diferente a la UFS del español o en el significado, o en la metáfora/imagen, o en ambos. Si la UF del chino es distinta de la de origen tanto en el significado como en la metáfora/imagen, utilizamos «la falsificación consciente» o «inconsciente» de Díaz Cintas (2003: 268) para describir esta situación. Es posible que contengan una nota en la pantalla. 
31 (iii) UF $\rightarrow$ No UF. En este caso, la traducción de la UF del español se lleva a cabo por el equivalente acuñado, la traducción literal, la amplificación, la explicitación y la sustitución situacional.

(a) El equivalente acuñado: se utiliza una expresión reconocida por el diccionario o por el uso lingüístico como equivalente en la lengua meta (cf. Molina 2006: 102). En este estudio, se utilizan como criterio el Nuevo diccionario español-chino (《新西汉词 典 ) y el Nueva era: Gran diccionario español-chino (《新时代西汉大词典》), los dos diccionarios más completos entre estas dos lenguas, para determinar si la traducción es reconocida por los diccionarios.

(b) La traducción literal: se traduce la UF a partir del significado de las palabras que la componen, sin tener en cuenta el significado de las UFS como unidad de sentido. Un ejemplo de esta técnica es traducir «llevar a la tumba» por «llevar algo en la tumba» (带入/带进坟墓, dairu/daijin fenmu).

(c) La amplificación: se añade información relacionada a la UF en español o se añaden notas en la pantalla para explicar el origen de la UF, etc. (cf. Molina 2006: 102).

(d) La explicitación: se explica el significado implícito de la UF. En esta técnica no hay cambio de significado. Por ejemplo, la traducción por ShenYing de la paremia «tirar la piedra y esconder la mano» es una explicitación, puesto que el segmento traducido es 做了坏事又装作事不关已 (zuo le huai shi you zhuangzuo shi bu guan ji), que significa «alguien hace algo mal y pretende que no tiene nada que ver consigo». (e) La sustitución situacional: se elimina el significado de la UF del español y se sustituye por una expresión distinta que se corresponda con el argumento ( $c f$. Pedersen 2011: 95). Esta técnica tiene un cambio destacable del significado, aunque no resulta incoherente en el contexto de lo que está sucediendo en la pantalla. Por ejemplo, en el T1E01, un personaje dice que el móvil va a «volverse loco». En esta situación, ShenYing traduce esta UF como «romperse» (坏掉, huaidiao), que tiene un significado diferente, pero que coincide con el argumento de la serie.

(iv) No UF $\rightarrow$ UF. Una No UF es traducido al chino como una UF.

(v) Omisión. Se omite la UF del español, es decir, no hay una UF ni una frase correspondiente a la UF del español. Raramente se utiliza en el fansubbing del español al chino.

\section{Metodología}

Como se menciona en el subapartado 1.2, los dos fansubs del MdT en China han sido realizados por los grupos ShenYing y YYeTs. En el presente estudio utilizamos el fansub de ShenYing como corpus principal y el de YYeTs como grupo de control para ver si ambos grupos traducen las UFS siguiendo la misma técnica de traducción.

De entre los 34 episodios de la serie, para este estudio seleccionamos los dos fansubs de 8 episodios de las tres temporadas, De la Temporada 3 solo consideramos el E22, dado que es el único episodio traducido de esa temporada por YYeTs. Los episodios seleccionados para el estudio se muestran en la Tabla 2. 


\begin{tabular}{|c|c|c|c|}
\hline & $\begin{array}{l}\text { Fansub de } \\
\text { ShenYing }\end{array}$ & $\begin{array}{l}\text { Fansub de } \\
\text { YYeTs }\end{array}$ & Duración temporal de los episodios \\
\hline Temporada 1 & \multicolumn{2}{|l|}{$\mathrm{E} 01, \mathrm{E} 02, \mathrm{E} 03, \mathrm{E} 08$} & $4 \mathrm{~h} 54 \mathrm{~min} \times 2=9 \mathrm{~h} 48 \mathrm{~min}$ \\
\hline Temporada 2 & \multicolumn{2}{|l|}{$\mathrm{E} 09, \mathrm{E} 10, \mathrm{E} 11$} & $3 \mathrm{~h} 29 \mathrm{~min} \times 2=6 \mathrm{~h} 58 \mathrm{~min}$ \\
\hline Temporada 3 & \multicolumn{2}{|l|}{$\mathrm{E} 22$} & $1 \mathrm{~h} 08 \mathrm{~min} \times 2=2 \mathrm{~h} 16 \mathrm{~min}$ \\
\hline \multicolumn{3}{|l|}{ EN TOTAL } & $19 \mathrm{~h} 02 \mathrm{~min}$ \\
\hline
\end{tabular}

Tabla 2: Episodios seleccionados para este estudio únicamente en las locuciones y las paremias y excluimos las colocaciones y las fórmulas rutinarias. Para facilitar el recuento de las técnicas de traducción, a continuación, presentamos los criterios seguidos para distinguir entre el equivalente acuñado y la traducción literal y entre la explicitación y la sustitución situacional. en el número de palabras que componen la UF en español y el reconocimiento de su traducción por los diccionarios o por el uso lingüístico. Cuando una UF en español contiene menos de tres palabras, y su traducción en chino aparece en los diccionarios o es reconocida por el uso lingüístico, lo consideramos a priori como un equivalente acuñado. Sirve de ejemplo la traducción de ShenYing de "gracias a» en el T1E08; esta locución se traduce como «por causa de alguien o algo que produce un bien o evita un mal» (多亏了, duokuile), es decir, se mantiene el significado. Además, la UF es reconocida por los diccionarios. En este caso, se considera un equivalente acuñado.

El cambio de significado de la UF determina fundamentalmente si la técnica es la explicitación o la sustitución situacional. Como se ha comentado anteriormente, la técnica utilizada en traducir la UF «volverse loco» es la sustitución situacional. Sin embargo, en el T1E08 se utiliza la explicitación para la misma UF, porque ShenYing lo traduce por «encantar a alguien» (超级追捧, chaoji zhuipeng) y YYeTs lo traduce por «ser fan» (很迷, hen mi).

\section{Resultado y análisis}

En este estudio, se encuentran en total 17 paremias y 358 locuciones. En la Figura 3 observamos que la omisión es muy poco utilizada. En el fansub de ShenYing, 26 UFS han sido traducidas como UFS mientras que en el de YYeTs, encontramos 24 casos en esta categoría. Además, una parte de las No UFS se han convertido en UFS en ambos fansubs (57 en el fansub de ShenYing y 61 en el fansub de YYeTs). Dentro de las No UFS, identificamos fórmulas rutinarias como «buen viaje» (traducido como «viento en popa en todo del camino», 一路顺风, yilu shunfeng), o frases como "no soy perfecto» (traducido como «no existe hombre perfecto», 人无完人, ren wu wanren). En ambos fansubs, aproximadamente el $90 \%$ de las UFS han sido traducidas como No UFS. 


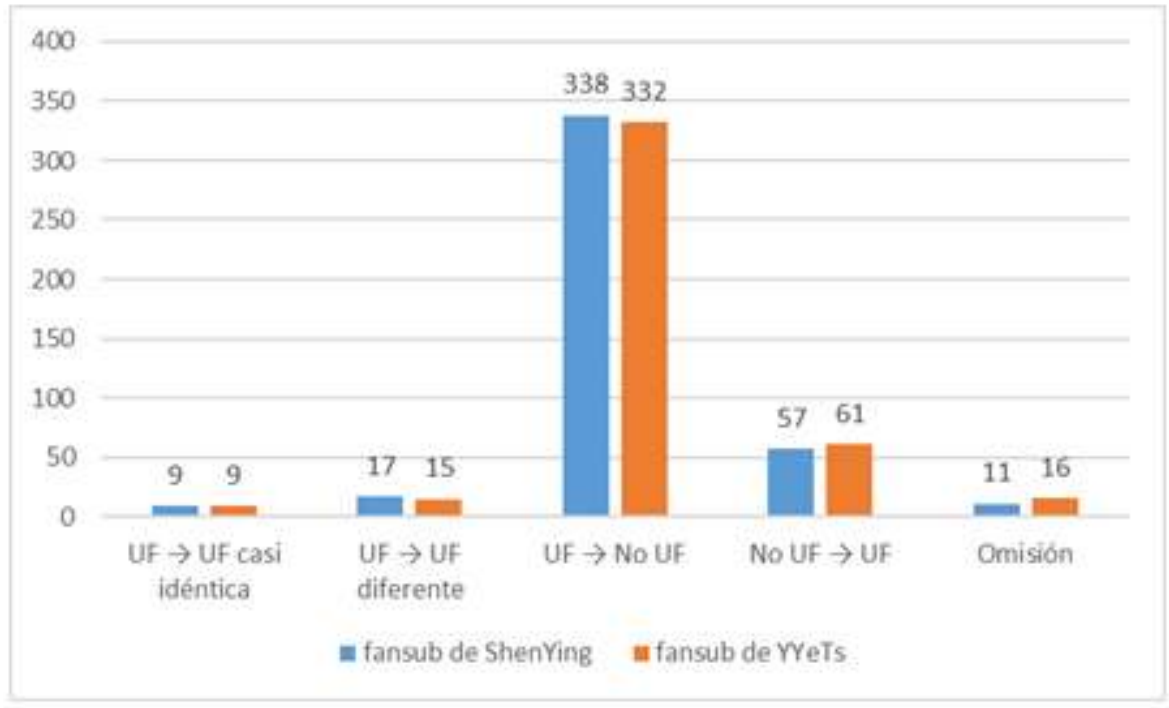

Figura 3: Las técnicas de traducción de las UFS en el fansub de ShenYing y el de YYeTs

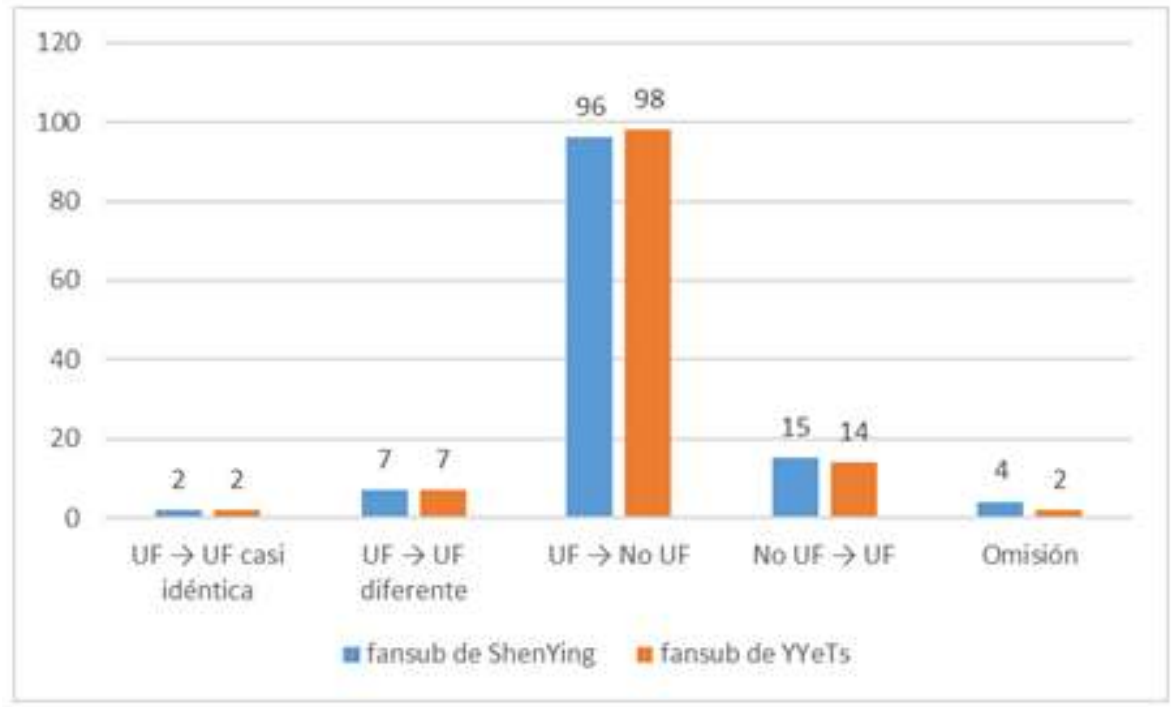

Figura 4: Las técnicas de traducción de las locuciones del T1E02, T2E09 y las 17 paremias del corpus

Nos centramos, a partir de este punto en las locuciones aparecidas en el T1E02, el T2E09 y en las 17 paremias del corpus para realizar un análisis más profundo. La Figura 4 muestra la distribución de las técnicas de traducción de las UFS en general. La Figura 5 recoge las técnicas de traducción en la categoría de la UF $\rightarrow$ No UF. La Figura 4 muestra que la mayoría de las UFS encontradas han sido traducidas como No UFS en ambos grupos. A partir de la Figura 5, observamos que en la categoría de la UF $\rightarrow$ No UF, las técnicas de traducción preferidas por los fansubbers son el equivalente acuñado y la explicitación. 


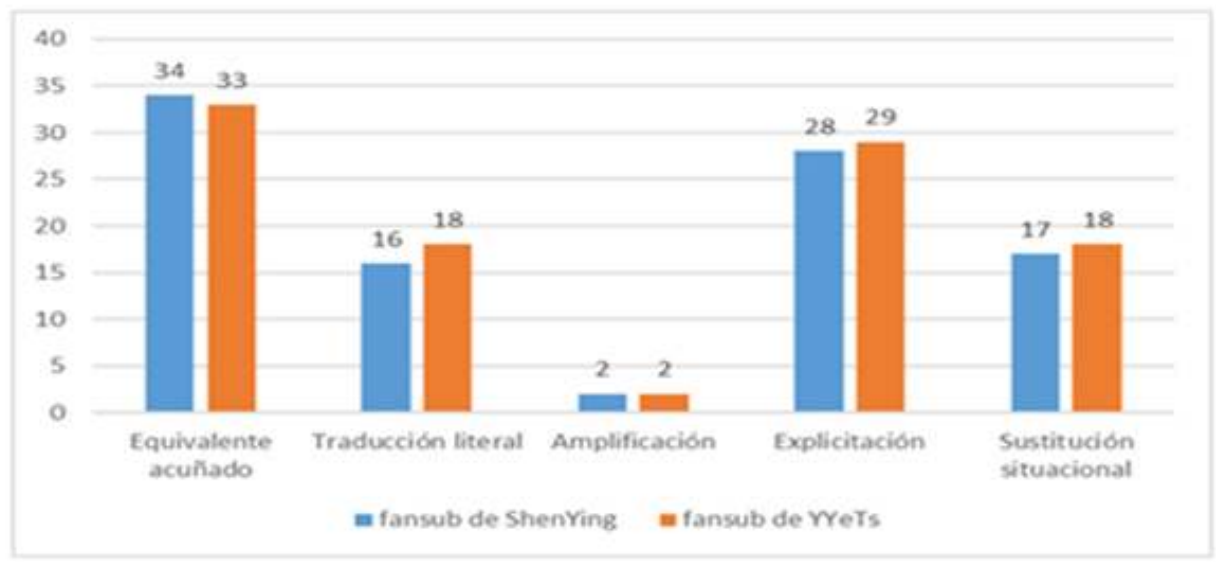

Figura 5: Las técnicas de traducción utilizadas en la UF $\rightarrow$ No UF

41 Después de presentar los datos cuantitativos, seleccionamos algunas locuciones de estos dos episodios y varias paremias como ejemplos del análisis cualitativo.

(a) UF $\rightarrow$ UF casi idéntica. Un ejemplo puede ser la traducción de ShenYing de «a su suerte». El fansub de ShenYing sustituye esta UF por el chengyu «a su suerte» (听天由 命, tingtian-youming). Consideramos una UF $\rightarrow$ UF casi idéntica, puesto que la UF en chino es un chengyu estilizado y no tiene una metáfora o imagen fuerte.

(b) UF $\rightarrow$ UF diferente. Dentro de esta categoría, se recogen las UFS diferentes en metáfora/imagen, pero con el mismo significado, como pueden ser la UF traducida de «ser una tumba» en el fansub de YYeTs, que es «cerrar la boca y no hablar, como si fuera una botella cerrada» (守口如瓶, shoukou-ruping). La UF en español y la UF en chino se diferencian en metáfora/imagen, pese al mismo significado de 'guardar rigurosamente un secreto'.

Además, se encuentran varias UFS diferentes tanto en metáfora/imagen como en significado, como pueden ser la UF expuesta en la Tabla 3. En el fansub de ShenYing, se utilizan el chengyu 一臂之力 (yibi-zhili) para sustituir la UFS en español. Este chengyu se combina con el verbo «ayudar» (助, zhu) y significa 'echar una mano a alguien'. Por ello, es evidente que es distinto a "poner al día». De acuerdo con el argumento de este episodio, Gil Pérez va a informar a los personajes de los detalles de la misión (salvar a López de Vega). No obstante, la UF traducida por ShenYing, si bien no tiene nada que ver con la UF en español, coincide con el contexto, porque en el resto de este episodio, este funcionario del Ministerio ayuda mucho a los personajes a cumplir su misión. Respecto al fansub de YYeTs, lo consideramos como una UF $\rightarrow$ No UF y es una sustitución situacional porque el segmento traducido no tiene relación con "poner al día». Lo interesante es que la traducción de YYeTs también se corresponde con el argumento, dado que los personajes llegan el día en el que Lope de Vega está en peligro a través de una puerta del tiempo. 


\begin{tabular}{|c|c|c|}
\hline \multirow{2}{*}{$\begin{array}{l}\text { UF y los subtítulos en } \\
\text { español }\end{array}$} & \multicolumn{2}{|c|}{ UF en chino y los subtítulos traducidos en chino } \\
\hline & ShenYing & YYeTs \\
\hline $\begin{array}{l}\text { [00:13:07] poner al día } \\
\text { El funcionario que los } \\
\text { recibirá se llama Gil Pérez. } \\
\text { Él los pondrá al dia. }\end{array}$ & 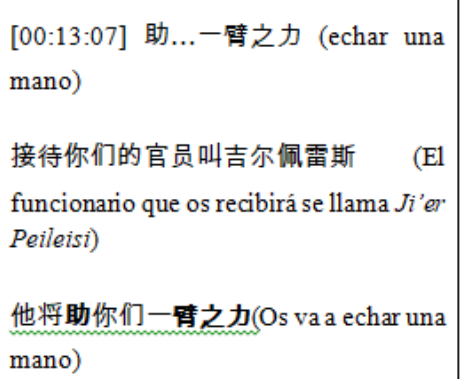 & $\begin{array}{l}\text { [00:09:10] 直接带到那一天 (llevar } \\
\text { directamente a ese día) } \\
\text { 接待你们的官员名叫希尔·佩雷斯 (E1 } \\
\text { funcionario que os recibirá se llama } \\
\text { Ji'er Peileisi) } \\
\text { 他会直接把你们带到那一天 (Os } \\
\text { llevará directamente a ese día) }\end{array}$ \\
\hline
\end{tabular}

Tabla 3: «poner al día», T1E02, ShenYing y YYeTs

Sin embargo, no todas las UFS diferentes pueden coincidir con el argumento de la serie con éxito. En el siguiente ejemplo (Tabla 4), ShenYing intenta otra vez la misma técnica y sustituye la paremia «le dijo la sartén al cazo» por el chengyu «habla el gallo al pato» (鸡同鸭讲, jitong-yajiang). Esta expresión proviene del cantonés y significa que dos partes no se pueden comunicar ni entender mutuamente, como si el gallo le hablase al pato. En el contexto, el personaje A quiere decir que el personaje B es igual que otro hombre y que también pone el trabajo por encima de todo. Por lo tanto, la UF en chino se contradice con el argumento y podrá causar problemas de entendimiento a los espectadores. Por su parte, YYeTs utiliza el geyan «quien huye 50 pasos se ríe de quien huye 100 pasos» (五十步笑百步, wushi'bu xiao baibu), que proviene de una obra clásica de la literatura china. Pese a mostrar una metáfora o imagen distinta, este geyan tiene el mismo significado que la paremia en español.

\begin{tabular}{|l|l|c|}
\hline \multicolumn{1}{|c|}{ UF en español } & \multicolumn{1}{|c|}{ UF de ShenYing } & \multicolumn{1}{c|}{ UF de YYeTs } \\
\hline $\begin{array}{l}\text { [00:09:35] “Le dijo 1a } \\
\text { sartén al cazo" }\end{array}$ & $\begin{array}{l}\text { [00:09:35]鸡同鹅讲 (habla el gallo al } \\
\text { pato) }\end{array}$ & $\begin{array}{l}\text { [00:10:00] 五十步笑百步 (quien huye } \\
50 \text { pasos se ríe de quien huye 100 pasos) }\end{array}$ \\
\hline
\end{tabular}

Tabla 4: «Le dijo la sartén al cazo», T1E08, ShenYing y YYeTs

(c) UF $\rightarrow$ No UF. Nos centramos primero en el equivalente acuñado. En el corpus, esta técnica es exclusivamente utilizada para la traducción de las locuciones. En ambos fansubs más del $84 \%$ de las locuciones que se traducen con esta técnica son las que contienen entre dos y tres palabras. Pese a que facilita la traducción, a veces esta técnica no es tan creativa como la sustitución con UFS y no ayuda a reconstruir el lenguaje de la serie original en los subtítulos traducidos. A diferencia de ShenYing, en la Tabla 5 YYeTs sustituye la UF en español por «ivaya a cualquier sitio fresco!», guanyongyu con el mismo significado. Aunque este guanyongyu es muy coloquial y se basa en una metáfora/imagen distinta, es interesante porque mantiene una mínima relación con la palabra «fresco». 


\begin{tabular}{|c|c|c|}
\hline \multirow{2}{*}{$\begin{array}{l}\text { UF y los subtítulos en } \\
\text { español }\end{array}$} & \multicolumn{2}{|c|}{ UF en chino y los subtitulos traducidos en chino } \\
\hline & ShenYing & YYeTs \\
\hline $\begin{array}{l}{[00: 24: 57] \text { ir con viento }} \\
\text { fresco }\end{array}$ & [00:24:57] 滚开 (jváyase!) & [00:21:28] 哪凉快滚哪去 (jvaya a cualquier \\
\hline $\begin{array}{l}\text { Esa dama estaba } \\
\text { conmigo, }\end{array}$ & $\begin{array}{l}\text { 这女人是跟我一起的 （Esta mujer } \\
\text { estaba conmigo） }\end{array}$ & $\begin{array}{l}\text { 这妞儿是和我一起的（Esta guapa estaba } \\
\text { conmigo) }\end{array}$ \\
\hline $\begin{array}{l}\text { asi que ya os podéis } i r \\
\text { con viento fresco. }\end{array}$ & 所以你给我滚开 (pues jváyase!) & $\begin{array}{l}\text { 你给我罗凉快滚表去 （pues ¡vaya a } \\
\text { cualquier sitio fresco!) }\end{array}$ \\
\hline Clasificación & equivalente acuñado & UF $\rightarrow$ UF diferente \\
\hline
\end{tabular}

Tabla 5: «ir con viento fresco», T1E02, ShenYing y YYeTs

En segundo lugar, observamos que la explicitación tiene mucha importancia en el fansubbing de las locuciones y paremias. A partir del corpus sabemos que más del $40 \%$ de las paremias se traducen al chino con esta técnica. Sirva de ejemplo la traducción de la paremia «hasta el rabo, todo es toro». En el T1E03, ambos grupos traducen esta UF como «es difícil de decir todo esto hasta el fin», que es el significado implícito de la paremia.

Otro buen ejemplo puede ser la traducción de ShenYing de la paremia «hay moros en la costa» (véase la Figura 6). En el T1E01, cuando los personajes están buscando algo en la habitación de un militar sin su permiso, se oye un ruido fuera de la habitación y parece que ese militar está a punto de volver. En ese momento, la doncella se agarra de la mano de la otra mujer y dice «ojito, que hay moros en la costa». El fansub de ShenYing lo traduce como «cuidado, debemos tener cuidado» (小心点 我们必须小心点, xiaoxin dian women bixu xiaoxin dian). A través de la explicitación, se elimina la imagen de la paremia y se mantiene su significado.

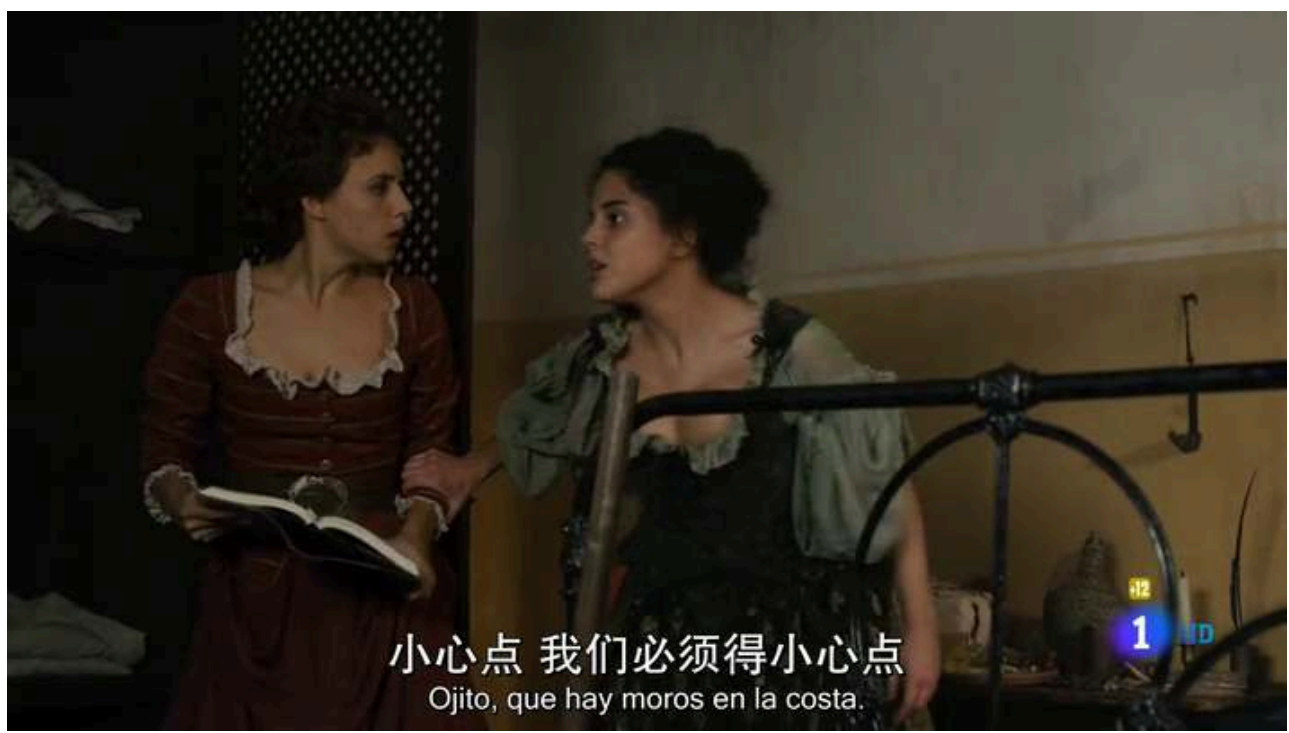

Figura 6: «hay moros en la costa», T1E01, ShenYing. Captura en 16/11/2018 
49

El último ejemplo que queremos comentar sobre la explicitación es la traducción de «a buenas horas, mangas verdes» (véase la Figura 7). Este ejemplo muestra cómo se combinan la explicitación y la amplificación. En la pantalla, Cervantes dice «A buenas horas, mangas verdes» a un hombre que pretende ser cuadrillero de la Santa Hermandad. La traducción inversa al español del subtítulo en chino es: «mangas verdes, llegas tarde otra vez». La traducción de YYeTs señala expresamente el significado de «llegar tarde» de la paremia y por ello se considera una explicitación. Aparte del subtítulo, YYeTs elabora una nota en la pantalla e indica que las mangas verdes son uniformes de la Santa Hermandad, una organización que siempre tarda un poco más de lo previsto en la investigación y detención.

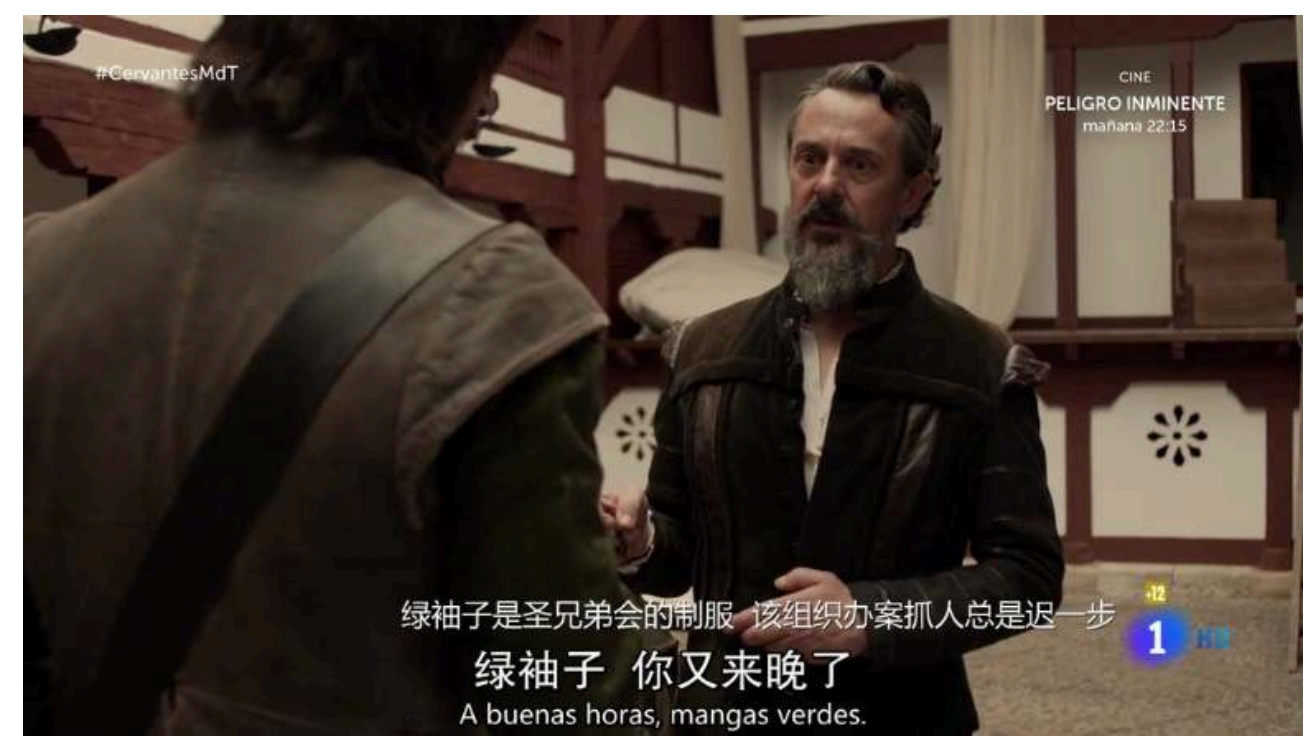

Figura 7: «A buenas horas, mangas verdes», T2E11, YYeTs. Captura en 16/11/2018

Aprovechamos la traducción de ShenYing de la misma paremia para comentar también el caso de la traducción literal en el corpus. ShenYing traduce esta UF como 恰当的时 机 圣兄弟会成员的身份, cuya traducción sería «la hora oportuna, la identidad del cuadrillero de la Santa Hermandad». Como se puede observar, ShenYing traduce esta UF a partir de las palabras sin tomar en consideración el significado de la UF como una unidad de sentido. Dado que el significado de una UF no es una suma simple del significado de cada palabra, con esta traducción se generan problemas de entendimiento en el subtítulo en chino.

51 Igual que la explicitación, la traducción literal se puede combinar con la amplificación. En el fansub de shenYing se encuentra esta combinación en la traducción de «si Mahoma no viene a la montaña, la montaña irá a Mahoma» (véase la Figura 8). En la pantalla se lee una nota libre, 《出自《古兰经》意指: 既然无法改变别人, 那就改变自 己», cuya traducción reversa al español es: «proviene del Corán, significa: como no se puede cambiar a los demás, nos cambiamos nosotros mismos». En el T2E10 del fansub de YYeTs, al traducir «Si la montaña no va a Mahoma, Mahoma irá a la montaña», junto con el subtítulo pone una nota entre corchetes: ““山不来找我 我便去找山” [伊斯兰谚 语]», cuya traducción reversa es: “"Si la montaña no viene a buscarme, yo voy a buscar la montaña" [refrán islámico]». 


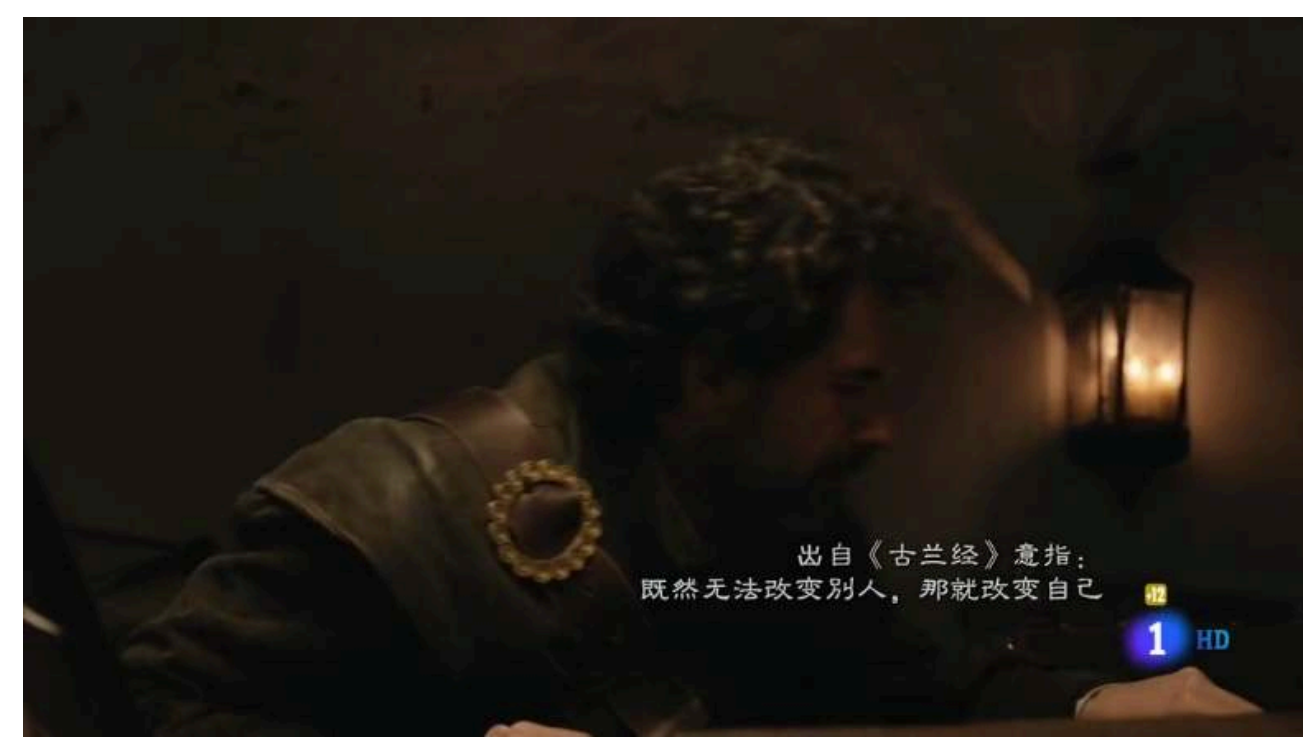

Figura 8: Combinación de la traducción literal y la amplificación, T1E02, ShenYing. Captura en $16 / 11 / 2018$

52 Cabe recordar que la amplificación también puede ser la adición de información relativa a la UF, como por ejemplo con «entrar como Pedro por Huesca» (cf. Tabla 6). ShenYing añade la información de «hacer lo que quiere», mientras que en el fansub de YYeTs, se añade el título de Pedro y el origen de esta paremia. Por lo tanto, ambos casos son amplificaciones.

\begin{tabular}{|l|l|l|}
\hline \multicolumn{1}{|c|}{ UF en español } & \multicolumn{1}{|c|}{ UF traducida por ShenYing } & \multicolumn{1}{|c|}{ UF traducida por YYeTs } \\
\hline $\begin{array}{l}\text { [00:39:18] entrar como } \\
\text { Pedro por Huesca }\end{array}$ & $\begin{array}{l}\text { [00:39:18] “像佩德罗进入韦斯卡一样 } \\
\text { 随心所欲” (“entra como Peideluo en } \\
\text { Weisika y hace lo que quiere") }\end{array}$ & $\begin{array}{l}\text { (e0:39:18] 佩德罗国王攻占韦斯卡的故事 } \\
\text { Weisika) }\end{array}$ \\
\hline
\end{tabular}

Tabla 6: «entrar como Pedro por Huesca», T3E22, ShenYing y YYeTs

53 Por último, hablamos de la sustitución situacional. Para explicar esta técnica, debemos volver a la paremia «hay moros en la costa». Para traducir esta UF, YYeTs utiliza la sustitución situacional y lo traduce por «viene alguien por fuera» (外面有人来了, waimian you ren laile). Esta traducción coincide con el argumento, en el que el militar francés está a punto de entrar a la habitación. Otro ejemplo es la traducción de YYeTs de la locución «poner al día» (cf. Tabla 3).

54 Asimismo, la traducción por ShenYing de la UF en la Tabla 7 también es un buen ejemplo de esta técnica. En este episodio, un personaje dice que su jefa sabe toda la historia, pero no la ha elogiado nunca para que «no se le suban los humos». En vez de explicitar el significado, ShenYing lo traduce como «no provocar líos», una expresión sin ninguna relación con la UF en español. Aun así, también se corresponde con el argumento de la serie (no quiere enfadar a su jefa). 


\begin{tabular}{|c|c|c|}
\hline \multirow{2}{*}{$\begin{array}{c}\text { UF y el subtítulo en } \\
\text { español }\end{array}$} & \multicolumn{2}{|c|}{ UF en chino y el subtítulo traducido en chino } \\
\hline & ShenYing & YYeTs \\
\hline $\begin{array}{l}\text { [00:21:59] Pero no se } \\
\text { lo digo para que no se } \\
\text { le suban los humos. }\end{array}$ & $\begin{array}{l}\text { [00:21:59] 但我什么都没说 为了不惹 } \\
\text { 事 (Pero no digo nada para no provocar } \\
\text { líos) }\end{array}$ & $\begin{array}{l}\text { [00:21:59] 但我怕她太舟橵了所以没和她 } \\
\text { 说过(Pero no quiero que ella sea demasiado } \\
\text { orgullosa/arrogante, por eso no se lo he } \\
\text { dicho nunca) }\end{array}$ \\
\hline Clasificación & sustitución situacional & explicitación \\
\hline
\end{tabular}

Tabla 7: «subírsele los humos», T2E09, ShenYing y YYeTs

(d) No UF $\rightarrow$ UF. En el corpus se localizan bastantes No UFS que han sido traducidas como chengyu o guanyongyu. En los T1E02 y T2E09 se encuentran varios ejemplos como los siguientes: «me dé por acabar lo que vos empezasteis» por el guanyongyu «resolver el lío causado por otra persona» (收拾烂摊子, shoushi lantanzi), traducir «sois adivina» por el chengyu «poder prever» (未卜先知, weibu xianzhi), traducir la palabra «mandan» por el chengyu «dar órdenes» (发号施令, fahao shiling), o traducir «plantar pepinos» como el guanyongyu «váyase a un sitio fresco» (一边凉快去, yibian liangkuai qu).

\section{Conclusión}

Los resultados del presente estudio profundizan el conocimiento sobre la traducción de locuciones y paremias en el fansubbing del español a chino. A partir del análisis realizado se puede sacar varias conclusiones.

En primer lugar, tras el análisis del corpus paralelo de ocho episodios del Ministerio del Tiempo, podemos observar que en general no hay una diferencia notable entre el empleo de las técnicas de traducción de ambos fansubs aunque frecuentemente utilizan diferentes técnicas para la misma unidad fraseológica. Siguiendo esta idea, destacamos la utilidad de un estudio de corpus paralelo en este tipo de trabajos basados en verificar las características en común entre los diferentes fansubs.

En segundo lugar, descubrimos que ) en el fansubbing del español al chino, la frecuencia del mecanismo de omisión es muy baja; ) no es común la sustitución de una UF en español por otra UF en chino (tanto si son UFS casi idénticas como si se trata de UFS diferentes), lo cual nos sorprende, porque el chino también es un idioma muy rico en refranes, frases hechas, etc.; ) la frecuencia de la técnica No UF $\rightarrow$ UF es bastante alta, especialmente si la comparamos con la frecuencia de las UF $\rightarrow$ UF; ) en la categoría de las UF $\rightarrow$ No UF, las técnicas de traducción preferidas por los fansubbers son el equivalente acuñado y la explicitación.

En tercer lugar, se pueden evaluar la creatividad de los fansubs y la reconstrucción del lenguaje de la serie de origen desde la perspectiva del uso de las UFS. El uso de las UFS en los subtítulos traducidos (incluso cuando no hay UFS en los subtítulos en español) permite compensar el desconocimiento de los espectadores de la lengua y cultura española. El uso frecuente de la UF $\rightarrow$ No UF sí fomentaría dicho desconocimiento.

Además de ilustrar la traducción de locuciones y paremias en el fansubbing del español al chino, el modelo de clasificación que hemos adaptado de la fraseología y de los Estudios de Traducción puede ser útil para otros investigadores en el futuro. Creemos 
que la catalogación en UFS y No UFS puede facilitar el recuento de las técnicas de traducción y la clarificación de datos.

61 Pese a la contribución que supone este estudio a varios ámbitos de investigación, cabe señalar las siguientes limitaciones: en primer lugar, falta una comparación más exhaustiva de la fraseología en español y la fraseología en chino. Debido a las diferencias entre la clasificación de las UFS en español y en chino, numerosas UFS, sobre todo las locuciones, han sido clasificadas como No UFS en chino, como pueden ser «jugarse la vida», «como si», etc. En el futuro, es necesario llevar a cabo más estudios para completar la correspondencia de las UFS españolas y las UFS chinas. En segundo lugar, dado que en el corpus el número de las paremias es muy limitado, sería útil replicar este estudio con otras series o películas para determinar si las conclusiones sobre las técnicas de traducción se pueden comprobar en otros corpus. En tercer lugar, pese a que hemos intentado establecer algunos límites de determinación entre las técnicas de traducción de la categoría de la UF $\rightarrow$ No UF, algunas técnicas que hemos indicado todavía pueden causar dudas. Asimismo, durante el análisis, encontramos diferentes tipos de errores en los dos fansubs, pero por limitaciones de tiempo y de espacio, no hemos incluido su análisis en este estudio. Este aspecto puede ser un punto de partida para futuros estudios dedicados a la identificación de los errores y la valoración de la calidad de los fansubs, por ejemplo, con el modelo FAR (Pedersen 2017). Para concluir, a pesar de ciertas limitaciones, este artículo proporciona perspectivas nuevas para varios ámbitos de investigación (en particular, para la fraseología y la subtitulación) y esperamos que aumenten los estudios que profundicen en este tema.

\section{BIBLIOGRAFÍA}

CORPAS PASTOR, Gloria (1997): Manual de fraseología española. Madrid: Gredos.

CUI, Jin-Yang (2017): El fenómeno del fansubbing en China : análisis comparativo entre la versión de fansubs y la profesional de la serie española El tiempo entre costuras. Trabajo de fin de máster. Bellaterra: Universitat Autònoma de Barcelona.

DELABASTITA, Dirk (1996): “Introduction”. The Translator 2(2): 127-139.

DÍAZ-CINTAS, Jorge (2003): Teoría y práctica de la subtitulación inglés-español. Barcelona: Editorial Ariel.

DÍAZ-CINTAS, Jorge y MUÑOZ SÁNCHEZ, Pablo (2006): "Fansubs: Audiovisual Translation in an Amateur Environment”. The Journal of Specialised Translation 6 (julio 2006): 37-52.

FERRER SIMÓ, María Rosario (2005): "Fansubs y scanlations: la influencia de los aficionados en los criterios profesionales". Puentes 6: 27-44.

HURTADO ALBIR, Amparo (2001): Traducción y traductología: Introducción a la traductología. Madrid: Cátedra. 
LAWICK, Heike van (2006): Metàfora, fraseologia i traducció: aplicació als somatismes en una obra de Bertolt Brecht. Aachen: Shaker.

MARCO, Josep (2009): "Normalisation and the Translation of Phraseology in the COVALT Corpus". Meta 54: 842-856.

MOLINA, Lucía (2006): El otoño del pingüino: Análisis descriptivo de la traducción de los culturemas. Castelló de la Plana: Publicacions de la Universitat Jaume I.

PEDERSEN, Jan (2017): “The FAR model: assessing quality in interlingual subtitling”. The Journal of Specialised Translation 28 (julio 2017): 210-229.

PÉREZ-GONZÁLEZ, Luis (2007): “Intervention in new amateur subtitling cultures: a multimodal account”. Linguistica Antverpiensia, New Series-Themes in Translation Studies 6: 67-80.

RAMíREZ BELLERÍN, Laureano (2004): Manual de traducción chino-castellano. Barcelona: Gedisa.

SEVILLA MUÑOZ, Julia, CRIDA ÁLVAREZ, Carlos Alberto (2013): "Las paremias y su clasificación".

Paremia 22: 105-114.

sun, Wei Zhang (孙维张), 1989. Hanyu Shuyu Xue (汉语熟语学) [La fraselogía china]. Changchun: Jilin Education Publishing House.

WANG, Dingkun (2017): "Fansubbing in China - With Reference to the Fansubbing Group YYeTs". The Journal of Specialised Translation 28 (julio 2017): 165-190.

WU, Fan (2014): La fraseología en chino y en español: caracterización y clasificación de las unidades fraseológicas y simbología de los zoónimos. Un estudio contrastivo. Tesis doctoral. Madrid: Universidad Autónoma de Madrid.

ZHANG, Leticia-Tian, CASSANY, Daniel (2019): "El fenómeno «danmu» y la participación mediática: Comprensión intercultural y aprendizaje de lenguas a través de «El Ministerio del Tiempo»”. Comunicar 27, n. 58: 19-29. https://doi.org/10.3916/C58-2019-02

\section{NOTAS}

1. Consulta Zhang y Cassany (2019) para más información sobre este fenómeno y los «danmu» del MdT.

2. Definición del Xiandai Hanyu Cidian (《现代汉语词典》, 2016, séptima edición). Traducción del chino de la autora.

\section{RESÚMENES}

El objetivo de este artículo es describir y analizar las técnicas utilizadas por los fansubbers en la traducción al chino de las locuciones y paremias de la serie española de televisión El Ministerio del Tiempo (MdT). Para alcanzar este objetivo introducimos el fansubbing y su desarrollo en China y la distribución de la serie MdT en dicho país. Más adelante, en el marco teórico, aclaramos los conceptos de las unidades fraseológicas en español, las unidades fraseológicas en chino y las técnicas de traducción. Adaptando varias propuestas, presentamos una clasificación breve de las 
técnicas de traducción de locuciones y paremias en el fansubbing del español al chino. Después, presentamos el corpus y la metodología, es decir, algunos criterios que se aplicaron en el recuento de las técnicas. En la parte de resultados y análisis, nos centramos tanto en datos que conseguimos tras el análisis cuantitativo como tras el cualitativo. Por último, exponemos conclusiones sobre este tema y evaluamos las contribuciones y ciertas limitaciones del presente artículo.

This paper aims to describe and analyze the techniques used by fansubbers in the chinese translation of the idioms and paremies from the Spanish TV series El Ministerio del Tiempo (MdT). In order to reach this objective, we introduce the concept of fansubbing and its development in China, and the distribution of the series MdT in said country. Later, we clarify in the theoretical framework the concepts of phraseological units in Spanish, phraseological units in Chinese, and translation techniques. Based on various proposals, we present a brief classification of translation techniques of idioms and paremies in the fansubbing from Spanish into Chinese. After that, we present our corpus and methodology, that is to say, the criteria applied for the scrutiny of the fansubbing techniques. In the results and analysis section, we focus on the data that we acquired from both the quantitative and the qualitative analysis. Finally, we draw out the conclusions on this topic and we evaluate the contributions and some limitations of the present paper.

\section{ÍNDICE}

Keywords: audiovisual translation, translation Spanish-Chinese, fansubbing, phraseological units, Spanish TV series

Palabras claves: traducción audiovisual, traducción español-chino, fansubbing, unidades fraseológicas, series de televisión españolas

\section{AUTOR}

PENG GAO

Universitat Pompeu Fabra Barcelona 